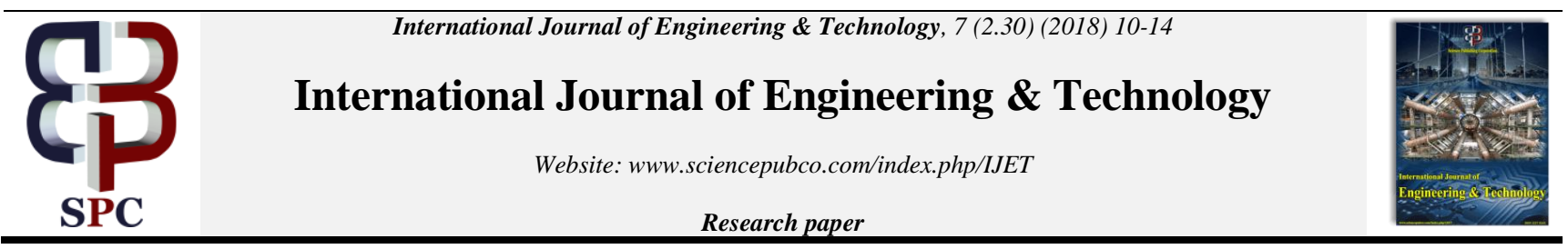

\title{
Big data analytic on block chain across healthcare sector
}

\author{
Miss. Shilpa ${ }^{1}$, Er. Rahul Sharma ${ }^{2}$, Mr. Sartaj Singh ${ }^{3}$ \\ ${ }^{1}$ Assistant Professor, School of Computer Applications, Lovely Professional University, Phagwara \\ ${ }^{2}$ Sr. Data Research Scientist, Gravity Code Lab Private Limited, India \\ ${ }^{3}$ Head of the Department, School of Computer Applications, Lovely Professional University, Phagwara \\ *Corresponding author E-mail: shilpa.21819@lpu.co.in
}

\begin{abstract}
Block chain is a decentralized transactional methodology for authorization and updates, in the Cryptocurrencies ecosystem. It is an exploring way in the Cryptocurrencies ecosystem to bit traditional corporate such as the mainstream healthcare and finance.

The responsive nature of healthcare data along the lasting provocation of inter-synchronization, healthcare info exchange and patient record matching has created opportunities for a Block chain to maintain the victory of the challenge.

The proposed paper aims to put Block chain technology or network based peer to peer authenticate layer on medical big data, for either information portability or set permission during third party involvement during some relevant big data analytic or findings. Based upon propose approaches, healthcare big data can be derived among multiple healthcare service providers, patients and analytic platforms with secure node to node distribution without any centralized ledger or storage point.
\end{abstract}

Keywords: Block Chain; Ecosystem; Decentralized; Cryptocurrencies; Miners; Bitcoin; Security; Interoperability.

\section{Introduction}

Now days, healthcare industries wants productive and efficient work from doctors and nurse staff as well that's a reason today healthcare industries are working on value-based business as compared to volume-based business. Value-based business will help to improve the healthcare practice and it will change the life style of everyone and help them in prevention of diseases, illness and infections and as a result it will be a reason for longer life for them. In past years, it was difficult to analyze the large amount of data because of complexity that become a reason for discovery of new technologies and mobile applications for early analysis. Therefore, in healthcare sectors Data analytics tools acts as important technology which helps in the management of complex and large amount of data that helps the industries to improve the medical practice for efficient work and accuracy in work flow.

But question is if data analytics tools are used in healthcare industries or organizations, how they are using that tool and what they will think in future and what challenges they will face during analysis with this tool?

Some of introductory model to define the healthcare intelligent network update even bit smarter with blockchain are given below:

\section{How The Change Healthcare Intelligent Healthcare Network Gets Even Smarter With Blockchain}

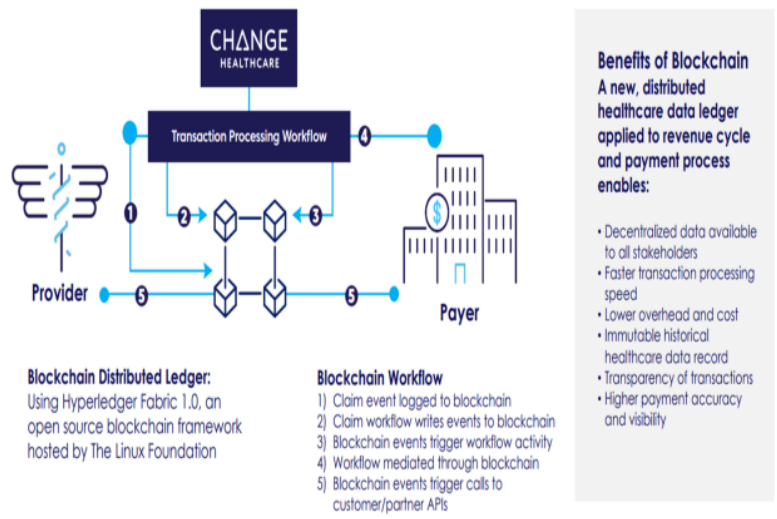

Fig. 1: Corporate Model for Healthcare Intelligent Network with Block Chain Technology.

\section{About health care data}

Due to big amount with complexity of healthcare private data it becomes more complex to control with the help of common and traditional data management methods, software and hardware.

In real time, healthcare data is analyzed based on the timeliness. Blood pressure and heart rate are best analysis for the real time healthcare analysis that helps for data generation.

Medical Data in healthcare sector can be categorized as follows:

\subsection{Genomic medical data}

Genotyping, DNA sequence and gene expression represents Genomic units that help in healthcare of patient. 


\subsection{Clinical inset data and clinical inset notes}

Around $80 \%$ of the clinical Data and Notes are required in healthcare which is in unstructured form also includes images and transcribed notes. (Yang et al., 2014):

1) Pre Structured Clinical data (EMR/HER data, Laboratory data)

2) Pre Unstructured Clinical data (Unstructured HER/EMR post-op notes, diagnostic test notes, medical images and graphical reports (X-ray or radiologic images), patient discharge detailed reports.

3) Pre Semi Structured Clinical data (Taking from other source based upon structure format), Patient Sentiment Clinical Data and Comportment Clinical Data.

4) Web based and social media based medical data such as Using Search Engines and Internet based networking sites like BlogSpot, LinkedIn, Facebook, twitter, healthcare or health plan web portals, portable devices i.e. Smartphone etc.

5) Mobility based streamed and electro sensor data.

\subsection{Health based research publication and clinical based reference data}

Health based drug or product information and Content based publications such as medical research articles or journals, clinical based research content and some medical based reference material.

\subsubsection{Business, executive and external source release data}

1) Financial sector data, billing documentary with scheduling and Insurance claims

2) Biometric machinery data such as handwritten, Iris based scans and Fingerprints etc.

\subsubsection{Other relevant and compatible data}

1) Portable or Fixed Device based data, patient suggestions or feedback and unfavorable events etc.

2) The content from release data portals or Personal Health Records messaging such as e-mails between the provider team and patient.

\section{About block chain technology}

Block chain is a futuristic secure distributed chain or system to stores private transaction records. It is in the form of centralized sharable, inflexible data of peer to peer transactions built from liner linked blocks and stored in the form of digital ledger.

Blockchain works same like database to stores information with only difference that the data is located in network of computers as data nodes where there is no central entity in the term of any federal or bank controlling the data.

\subsection{Benefits of block chain technology for big data ana- lytic in health care applications}

Effective Analysis can be performed easily on heterogeneous data, which is collected from various different sources.

For Example: For Proper health view of patient, patient data is properly analyzed with the help of internet and also by reading the patient health with the help of various measurement meters such as accelerometers, heart meters, glucose meters.

It became easy to identify the effective treatment for patient by applying advanced analytics on individual for prevention and lifestyle change.

However, due to some cyber attacks on private healthcare data, there are number of limitations are existing. To reduce data leakage during healthcare operations or transactions as big data analytic, Blockchain is a best fit technology to provide peer to peer healthcare data protection as authenticate framework. Some most highlighted benefit of Blockchain technology on big data analytic is:

1) Store private medical data on peer to peer decentralized system as big data form and utilize this data for analytic purposes on immediate request.

2) If sometime big data is crash on one of the network or system, it can be immediate recovered through other system as decentralized block nodes recovery.

3) If any patient wants to share private data with direct hospital staff, it will be work as secure transaction without any involvement of third party database or ledger.

4) If one healthcare industry wants to share some limited and private data with other healthcare industry without any third party or data broker involvement for data analytic purposes to conclude any serious problem output, Blockchain is a way to execute it without any worry between parties.

Some of the blockchain promising use cases for healthcare industry are given below:

\section{Blockchain Technology - Promising Use Cases for Healthcare Industry}

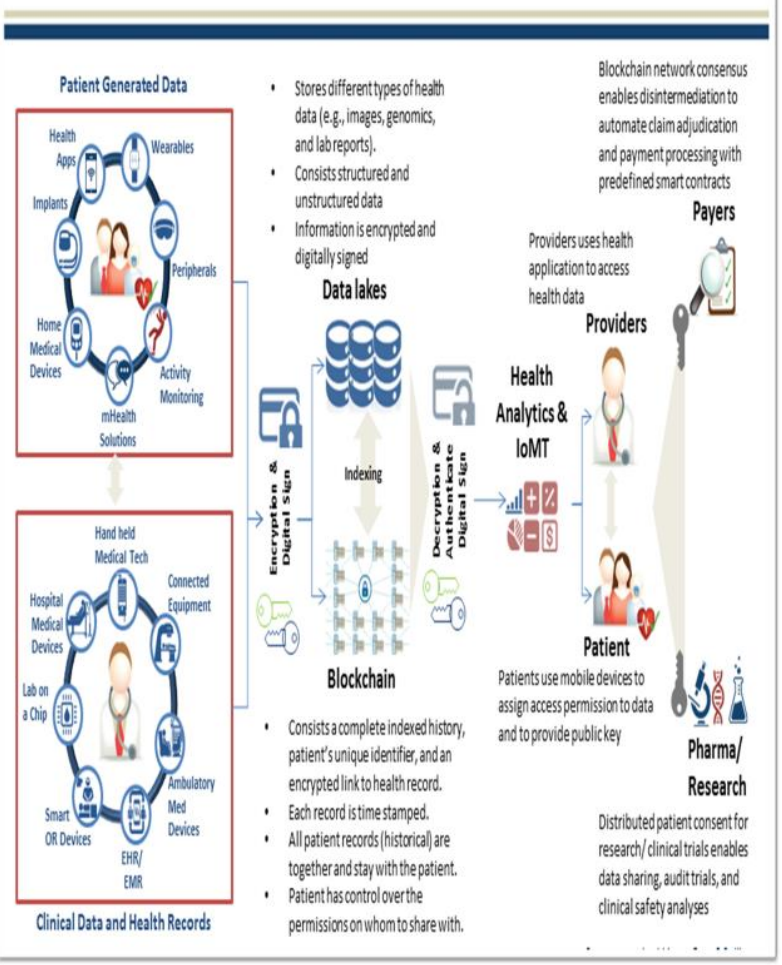

Fig. 2: Block chain Promising Use Cases for Healthcare Industry.

\section{Reviewed methodology}

\subsection{Matthew herland, randall wald and taghi $m$ khosh- goftaar (2014)}

in this paper author is discussing about the tools and approaches which are used for analysis of healthcare data which is gathered from different sources. They are discussing how data is gathered from different sources to gain the knowledge about the patient and healthcare services. They are discussing about the various tools and methods which are used to analyze condition of patients whether they will be released from ICU and which treatment will be beneficial for them for better response.

\subsection{Wullianallur raghupathi, viju raghupathi (2014)}

In this paper author discussing about the big data analytics benefits in healthcare. In this, they have taken a healthcare survey of U.S having more than 2700 members that contains the data about patient, clinical reports and other survey. Based on which it be- 
come easy to analyze the data and making decisions. In this conceptual model is used to analyze the data which is collected from different sources. They are discussing about various challenges that can be improved with latest enhancement.

\subsection{Mohammad ahmad alkhatib, amir talaei-khoei, amir hossein ghapanchi (2015)}

In this paper author is discussing about the various analysis technologies in healthcare data analytics that are improving the healthcare performance in many areas and with healthcare tools , large amount of data is easily managed. During analysis various challenged are faced how they can be improved for better result.

\subsection{Lidong wang and chery ann alexander (2015)}

Author discuss about the benefits of Big data in healthcare and medical, how we can analyze the patient data, how we can predict the outcome and what are the challenges occurring in big data analysis. Main focus of this paper is of medical sensor data streaming, processing and analysis of medical data. Hadoop-Map Reduce Platform is used to analyze the patient old data to determine the diseases. They are discussing about the proposed model for patient analyze and providing treatment for better result.

4.5. Javier andreu-perez, guang-zhong yang, robert $D$. merrifield, stephen T. C. wong, carmen C. Y. poon (2015)

in this paper author discussing about the development of Big Data in healthcare and medical field. With the rise in Big data, also privacy, security challenges are rising. They are discussing recent activities and opportunities in future and how big data processing is used in bioinformatics and health informatics etc. They are discussing the use of various tools and techniques which are used to sense the human data for analyze and providing better treatment for better result.

\subsection{Shrutika dhoka, R. A. kudale (2015)}

In this paper, they proposed a referential architecture on spark platform to overcome the issues of healthcare data processing. The architecture is composed of various components that are used for analyzing fast growing chronic illness of people. The main focus of this paper is mining of data of health record using proposed architecture.

\subsection{Asaph azaria, ariel ekblaw, andrew lippman and thiago vieira}

In this paper author discussing about propose system MedRec which is a novel that helps for decentralization of record management system for handling EMRs using Blockchain. Patients can get easily access of medical information from treatment sites and health access providers using propose system. In handling of sensitive information of patiens, Propose MedRec helps in managing patient authentication, unique leveraging properties of blockchain, confidentiality of patient data and data sharing are crucial considerations. Author discussing that all the researchers, public authorities and medical stakeholders should participate in the blockchain network as "miner" for making the system convenient and adaptable.

\subsection{Sanskruti patel and ATUL patel (2016)}

Author discussing about the implementation of big data solutions on healthcare sector by which meaningful and knowledgeable information is obtained. Author discussing about the Big Data Analytics with challenges, threats in healthcare sector and various opportunities in healthcare. By Big data solutions, it becomes easy to save patient life and provide various services to patients.

\subsection{Prasan kumar sahoo, suvendu kumar moha- patra,and shih-LIN WU (2017)}

In this paper author discussing about patient health condition after prediction of various parameters. They proposed a stochastic model to predict the health of patient. Mapreduce framework model is proposed to reduce the workload.Probabilistics data acquisition model is proposed for cloud healthcare data. They are discussing that in future new platform will be proposed for real time analysis of healthcare data.

\subsection{Choong HO LEE, hyung-JIN YOON (2017)}

Author discussing about various premises and challenges in medical big data analysis in various areas. They are introducing the issues related with medical big data like missing values, bias control and other data sharing issues. They are discussing about the various data mining algorithm used for mining of medical data that are used to gaining the knowledge from the patient data to predict the disease. They are discussing about the issues in medical data like scarce, data quality, data inconsistency. Author discussing that due to these challenges it is difficult to reduce the waste of resources and increase the performance of the healthcare data.

\subsection{Mehdi benchoufi, philippe ravaud}

In this research article, Authors explore the blockchain functionalities that are applied to medical or clinical demo and they elaborate and illustrate concretely its standard theory in the reference of assent to a demo based trial protocol. Authors are trying to highlight the potential impact of Blockchain technology in the setting of medical or clinical trials will shed innovative light on how modern trial methods could develop and avail from Blockchain platform to tackle the existing provocation.

\subsection{Tsung-ting kuo, hyeon-EUI KIM, lucila OHNO- machado}

In this paper, Authors discussing about Base Cryptocurrencies i.e. Bitcoin and the highlighting Blockchain platform to provide decentralized technology, an inflexible audit trail, robustness, security \& privacy and data provenance. Authors contrasted Blockchain platform among technology such as Blockchain Web 1.0, Web 2.0 and 3.0 and explored the some major benefits of Blockchain compared to existing distributed databases for medical or biomedical applications and provided the latest biomedical/health care applications overview of Blockchain such as enhance insure claim process, biomedical research, proposed medical record management, and advanced medical or health care private data ledger. Authors elaborate major challenges to adopting blockchain technology in the term of scalability, the threat of $51 \%$ cyber attack and confidentiality.

\subsection{J. Archenaa and Dr E. A.mary anita (2017)}

in this paper author discussing about the model which is proposed for the medical data analysis regarding diabetes. They are developing healthcare recommendation engine using big data analytics which is used to predict the patient conditions by various factors of patients. Author discussing that Apache spark is powerful for analysis of medical data and Bayesian method is effective method for data analysis. Prediction model is developed to predict the patient using different cases using classification.

4.14. Karim abouelmehdi, abderrahim beni-hessane and hayat khaloufi (2018) 
in this paper author is discussing about the risk in big data healthcare and introducing latest technologies to handle these risks. They are discussing the healthcare data analytics security in three matters: Data security, Access Control and information security. Yazan et al. discussing about the Data security lifecycle and addressing the threats and attacks in each phase of lifecycle and then they have proposed a information security lifecycle model to monitor and analyze the patient data with security. In this paper author discussing about the big data analysis challenges in Healthcare industry. They are discussing the strength and limitation of proposed methods of healthcare, how they are beneficial in future trends.

\subsection{World economic forum annual meeting (2018)}

in this meeting it is discussed that now a days in digital world, patients are also treated digitally that provide holistic solution for improvement and reducing illness. Various new technologies are used to deal with patients who help for success in future but not face challenges in medical data analysis. Everyone in digital world understanding digital ecosystem of healthcare.

\section{Proposed work}

The following proposed model is represented as logical architecture to implement Blockchain technology on healthcare big data for analytic purposes during medical activities and enhancement:

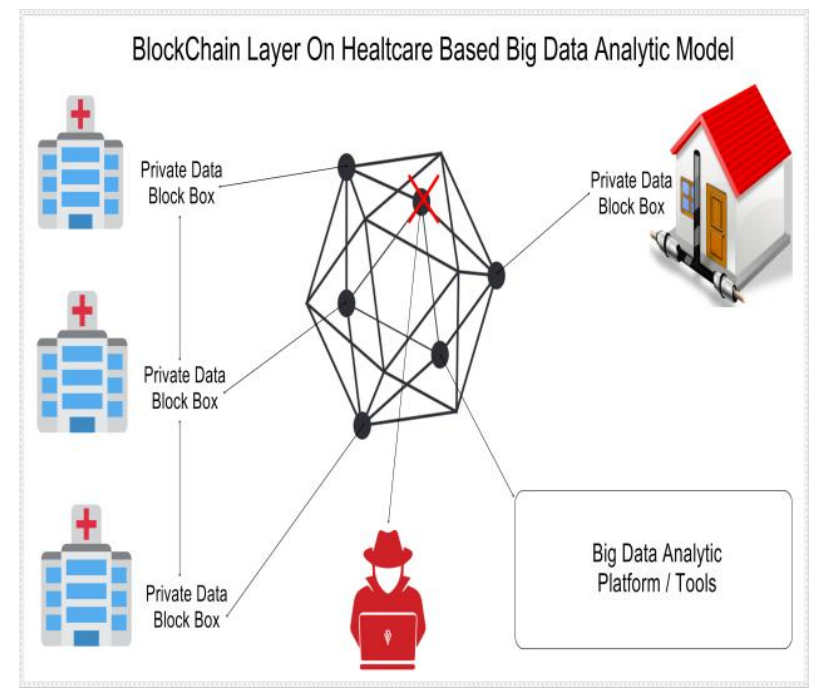

Fig. 3: Proposed Considerations Architecture Model.

\section{Propose approach execution}

1) Creating trusted ecosystem for decision making

Propose Blockchain approach has ability of using time stamp that provide the authentication for changes in dataset that provides the permissions to more than one user for editing a document for proper management of EHR data.

With the help of propose technology, patients can share their information of their secret with their new members and can exchange between the providers. In this way, patients as well as providers can share their records with each other sacredly based on which they can verify the propose record and can accept or reject the data that helps in evolution of new records.

In addition to renovate medical or heath based patient agency and control among private data exchanging, this approach may useful to reduce or remove one of the major barriers to medical decisionmaking in the term of the lack of ability to bond the private data obtainable at the point of care.

2) Enable Patient centralized interoperability and health information as portability exchange
Blockchain ecosystem may smooth under issues of patient consent for data exchange that is the one of the major obstacles of developing a health information exchange system.

Through this approach, Patients are act like a major to give consent statements at any point during their care journey to confident with the Blockchain to hold them securely. Healthcare professionals can act upon those directives, and the systems that they use can interpret them as access control decisions - with the assurance that the system is adhering to patient wishes.

Without patient as well as healthcare organization consent, analytic platform or mechanisms will denied to control information under big data strategy. After consent, they will be able to make findings for medical enhancement.

3) Enabling Recovery Points at any stage during health information exchange and system crash state

Using propose approach, on every state, some relevant recovery points and enabled in the form of transactions or system crash.

If one system will transfer or enable permission during any private information to other system and on any particular point, failure will occur, state will be automatically rollback and failure entry will be updated on each node of distributed systems as peer ledger. Moreover, if any attacker try to shake this transaction state, will get failure or rejection only because of multi system conditions verification.

On other hand, if any system data will be crash for some hidden reason, all other blocks or distributed ledger will update and recover data in auto state without any data lose identity.

4) Enabling Secure Conditional Authentication Layer across multiple data access points

Using propose approach, each transaction will be done after conditional authentication computation i.e. on the basis upon all other blocks similar ledger matching tabular form. If one of the distributed block or data node will not match with other decentralized blocks, transaction will go on failure state and attacker or unauthenticated user will denied to proceed further on system.

\section{Conclusion and future work}

Today, most of the healthcare data is access by network points with any security or privacy. All the private data like patient information, disease description, and healthcare accountable measures are not safe. Even healthcare big data controls by big data platform/tools for analytic purposes are also breaking some security measures. During some existing issues, Blockchain is a best fit model as security measures on peer to peer data accessibility with permission and management process.

In this paper, we propose a Blockchain model for healthcare big data analytic purposes. This model is divided into number of approaches to elaborate benefit and positioning under propose research work. Using propose approaches, we can reduce the big data leakage or accessibility against third party cyber attack on healthcare sector.

In future, we will propose a platform for healthcare data analytic and finding statements for better enhancement or outcomes.

\section{References}

[1] Wullianallur Raghupathi, Viju Raghupathi. (2014). big data analytics in healthcare: promise and potential. Raghupathi and Raghupathi Health Information Science and Systems 2014.

[2] Karim Abouelmehdi, Abderrahim Beni-Hessane and Hayat Khaloufi. (2018). big healthcare data: preserving security and privacy. Journal of Big Data. Department of Computer Science Laboratory LAMAPI and LAROSERI, Chouaib Doukkali University, El Jadida, Morocco.

[3] Mohammad Ahmad Alkhatib, Amir Talaei-Khoei and Amir Hossein Ghapanchi. (2015). Analysis of Research in Healthcare Data Analytics. In Proceedings of Australasian Conference on Information Systems, Sydney (2015).

[4] Sanskruti Patel and Atul Patel. (2016). A B S Ig Data Revolution in Health Care Ector: Opportunities, Challenges and Technological 
Advancements. In Proceedings of the International Journal of Information Sciences and Techniques (Ijist) Vol.6, No.1/2, March 2016.

[5] Ashwin Belle, Raghuram Thiagarajan, S. M. Reza Soroushmehr Fatemeh Navidi, Daniel A. Beard and Kayvan Najarian. (2015) Big Data Analytics in Healthcare. BioMed Research International, Volume 2015, Article ID 370194. Hindawi Publishing Corporation.

[6] Lidong Wang and Cheryl Ann Alexander. (2015). Big Data in Medical Applications and Health Care. Current Research in Medicine 2015, $6(1): 1.8$.

[7] Big Data Technologies in Healthcare- Needs, opportunities and challenges. Big Data Value Association. (2016).

[8] Paola Cerchiello and Paolo Giudici. (2016). Big data analysis for financial risk management. Open access Journal of Big Data.

[9] Liang Y1 and Kelemen A. (2016). Big Data Science and its Applications in Healthcare and Medical Research: Challenges and Opportunities. Austin Biom and Biostat - Volume 3 Issue 1 - 2016. Austin Publishing Group, U.S.A.

[10] Asaph Azaria, Ariel Ekblaw, Thiago Vieira and Andrew Lippman. (2016). MedRec: Using Blockchain for Medical Data Access and Permission Management. In proceedings 2nd International Conference on Open and Big Data (2016). IEEE Computer Society.

[11] Mehdi Benchoufi and Philippe Ravaud. (2017). Blockchain technology for improving clinical research quality. Benchoufi and Ravaud Trials (2017). BioMed Central.

[12] Matthias Mettler. (2016). Blockchain Technology in HealthcareThe Revolution Starts Here. In Proceedings of the IEEE 18th International Conference on e-Health Networking, Applications and Services (Healthcom).

[13] Tsung-Ting Kuo, Hyeon-Eui Kim and Lucila Ohno-Machado. (2017). Blockchain distributed ledger technologies for biomedical and health care applications. In proceedings of the Journal of the American Medical Informatics Association, 24(6), 2017, 12111220. Advance Access Publication, OXFORD. 